PHYSICAL REVIEW E 93, 069901(E) (2016)

\title{
Erratum: Dynamics of an electric dipole moment in a stochastic electric field \\ [Phys. Rev. E 88, 022127 (2013)]
}

\author{
Y. B. Band
}

(Received 19 May 2016; published 6 June 2016)

DOI: 10.1103/PhysRevE.93.069901

Equation (3) in the paper should read

$$
0=\frac{\partial \mathcal{L}}{\partial \theta}-\frac{d}{d t} \frac{\partial \mathcal{L}}{\partial \dot{\theta}} \Rightarrow \ddot{\theta}+\frac{E d}{I} \sin \theta-\frac{\omega^{2}}{2} \frac{\sin 2 \theta}{\sin ^{4} \theta}=0 .
$$

The factor of $\sin ^{-4} \theta$ in the last term on the left hand side of the differential equation was incorrectly written as $\sin ^{-2} \theta$, and this factor was left out of the terms proportional to $\omega^{2}$ in Eqs. (5) and (7). This correction does not affect the rest of the paper since the correct expressions were used in the calculations. 